This item was submitted to Loughborough's Research Repository by the author.

Items in Figshare are protected by copyright, with all rights reserved, unless otherwise indicated.

\title{
Finite element simulation of lamellar copper-silver composites
}

PLEASE CITE THE PUBLISHED VERSION

http://dx.doi.org/10.1016/j.commatsci.2015.01.012

\section{PUBLISHER}

(c) Elsevier

\section{VERSION}

NA (Not Applicable or Unknown)

\section{PUBLISHER STATEMENT}

This work is made available according to the conditions of the Creative Commons Attribution-NonCommercialNoDerivatives 4.0 International (CC BY-NC-ND 4.0) licence. Full details of this licence are available at: https://creativecommons.org/licenses/by-nc-nd/4.0/

\section{LICENCE}

CC BY-NC-ND 4.0

\section{REPOSITORY RECORD}

Dodla, Srihari, A. Bertram, and M. Kruger. 2019. "Finite Element Simulation of Lamellar Copper-silver Composites". figshare. https://hdl.handle.net/2134/19013. 


\begin{abstract}
The mechanical behaviour and texture evolution of lamellar $\mathrm{Cu}-\mathrm{Ag}$ polycrystals are numerically investigated for a uniaxial compression test by three dimensional finite element simulations. In the representative volume element (RVE), the lamellar structure is generated inside the grains. A crystal plasticity material model for large deformations is used at each integration point. In this work, two cold drawn textured $\mathrm{Cu}-\mathrm{Ag}$ polycrystals are modeled by periodic Voronoi tessellations in the finite element (FE) software ABAQUS. The FE calculations use periodic boundary conditions to simulate the mechanical behavior of the textured polycrystals. The numerical model is validated by experimental compression tests for a constant strain rate of $10^{-4} s^{-1}$ at room temperature. The numerical results in terms of texture of each phase and the mechanical behavior have been compared with the experimental results.
\end{abstract}

Keywords: Two-phase $\mathrm{Cu}-\mathrm{Ag}$ polycrystals, lamellar phase structure, crystal plasticity material model, RVE, crystallographic texture

\title{
1. Introduction
}

Two-phase metals are widely used in magnetic science and technology, automobile and aerospace industries, since they can show high strength, high hardness and sufficient ductility [40], [18], [41]. Two-phase $\mathrm{Cu}-\mathrm{Ag}$ composites are used as conductor materials for the application in the magnets $[40,2,18,41,13]$. With the increase of $\mathrm{Ag}$ content in the $\mathrm{Cu}-\mathrm{Ag}$ composite, i.e., for an eutectic composition $\mathrm{Cu}-72 \mathrm{wt} \% \mathrm{Ag}$, the strength is increased by the formation of alternate layers of $\mathrm{Cu}$ and $\mathrm{Ag}$ thus forming a lamellar structure inside the material [16, 19]. Several procedures are available for fabricating such materials including different types of ingot metallurgy [8, 20, 21], and powder metallurgy [5]. The eutectic (lamellar) two-phase polycrystals have shown unique mechanical properties, which are beyond the characteristics of their individual phases [36], [8], $[15]$.

$\mathrm{Cu}$ and $\mathrm{Ag}$ have the same lattice structure (face centered cubic) and both have the same slip geometry. Therefore various $\mathrm{Cu}$ and $\mathrm{Ag}$ alloys can be cold deformed to produce the fine $\mathrm{Cu} / \mathrm{Ag}$ lamellae [40]. During the cold drawing operation there is a significant refinement in the lamellar structure which consequentially results in increased strength and strain [6]. During the deformation process of such lamellar $\mathrm{Cu}-\mathrm{Ag}$ polycrystals, the initial microstructure, which includes the grain size and morphology, the lamellae width and distance between the adjacent lamellae and the orientations of each phase/grain are crucial for the determination of the effective mechanical behavior. The $\mathrm{Cu} / \mathrm{Ag}$ lamellae interactions and the volume fraction of each phase are also essential since they influence the effective mechanical behavior. In metal forming processes, forces are applied to the work piece such that the induced compressive stresses in the material are greater than the yield point stress and less than the ultimate stress. Therefore the material undergoes inhomogenous plastic deformations which are used for changing the shape of the material. To

*Corresponding author. Tel. +4939167 52246; Fax +49 391672863

Email address: srihari.dodla@st.ovgu.de (Srihari Dodla) 
understand and identify such complex deformation behavior various multiscale approaches have been developed. However, the microstructures consisting of two phases with an eutectic structure occur in many materials of technological interest (Ti-Al, $\mathrm{Cu}-\mathrm{Ag}, \mathrm{Zr}-\mathrm{Nb}$, etc). The strength of such eutectic microstructure consisting of a fine lamellar structure show higher strength than expected from a simple rule of mixture [40]. The crucial questions for modeling two phase lamellar structures are: How do the lamellae orientations of the individual crystallite relate to the lattice arrangement of the grain, and how to combine three scales (lamella scale, grain scale and macro scale) to render the macroscopic properties of two phase polycrystals. The answers to these questions are of interest for the design of lamellar composites and for the prediction of the mechanical behavior and texture of two phase polycrystals. The mechanical behavior and texture evolution of single phase metals are addressed in many studies. Crystal plasticity models have been chosen for calculating the texture evolution by using the Taylor model [30, 31, 32, 42] and self-consistent modeling $[34,35]$. Finite element method with implemented crystal plasticity model has been used to capture the grain interactions $[30,39]$. In the case of two phase composites, the presence of the second phase can also effect the texture evolution. A viscoplastic self-consistent polycrystal model has been applied by [8] to study the texture development of lamellar $\mathrm{Cu}-\mathrm{Ag}$ composites, where the two phase polycrystal simulations are compared with the corresponding experimental measurements. However, the disadvantage of this approach is that the morphology of the lamella cannot be modeled inside the grain. Texture evolution during non-crystallographic shear banding in a plane strain compressed $\mathrm{Cu}-\mathrm{Ag}$ composite using crystal plasticity finite element simulations has been recently studied by $[27,28]$. However, the effect of the lamellar morphology on the polycrystal simulations is still not considered.

In the present work, we model the lamellar two phase polycrystal considering three different scales information of three different scales (lamella scale, grain scale, and macroscale). Two scales (lamella and grain scale) are combined, i.e., amplifying the lamella scale to fit the grain scale. Three dimensional finite element simulations of two cold drawn $\mathrm{Cu}-\mathrm{Ag}$ samples of diameter $d_{1}=12.42 \mathrm{~mm}$ and $d_{2}=6.73 \mathrm{~mm}$ have been modeled. Initially both samples have a certain crystallographic texture, and the texture information of these two samples by bulk XRD measurements has been given in the literature [14]. The article is structured as follows. In Section 2, experimental observations of both samples are introduced. Section 3 deals with the constitutive equations of a finite elasto-viscoplastic material model based on the isomorphy concept of the elastic laws [7]. Section 4 focuses on the finite element simulation of the lamellar composites by generating the lamellar structure inside the grains. Section 5 explains the validation results in terms of stress-strain behavior and texture. Section 6 discusses the results and gives conclusions. Notation. We use the symbolic notation given in the continuum mechanics text book Bertram [7]. Scalars, vectors, second-order and fourth-order tensors are denoted by $a$, a, A, and A, respectively. The scalar, dyadic, and Rayleigh product are given by $\cdot, \otimes$, and $*$, respectively, where $\mathbf{a} \cdot \mathbf{b}:=a_{i} b_{i}, \mathbf{a} \otimes \mathbf{b}:=a_{i} b_{j} \mathbf{e}_{i} \otimes \mathbf{e}_{j}, \mathbf{A} * \mathbb{C}:=C_{i j k l} \mathbf{A} \mathbf{e}_{i} \otimes \mathbf{A} \mathbf{e}_{j} \otimes \mathbf{A} \mathbf{e}_{k} \otimes \mathbf{A} \mathbf{e}_{l}$. : denotes the double contraction between tensors, i.e., $\mathbf{A}: \mathbf{B}:=A_{i j} B_{i j} . \mathbf{A}^{T}, \mathbf{A}^{-1}$ and $\dot{\mathbf{A}}$ denote the transpose, the inverse, and the material time derivative of a second-order tensor $\mathbf{A}$. The linear mapping of a second-order tensor $\mathbf{A}$ by a fourth-order tensor $\mathbb{C}$ is written as $\mathbb{C}[\mathbf{A}]$.

\section{Experiments}

\subsection{Drawn Samples}

The two cold drawn $\mathrm{Cu}$-Ag rods having diameters of $12.42 \mathrm{~mm}\left(d_{1}\right)$ and $6.73 \mathrm{~mm}\left(d_{2}\right)$ are produced by die casting. Samples $\left(d_{1}, d_{2}\right)$ are sectioned in both longitudinal and transverse directions. The sectioned samples are grinded, polished, etched and examined in a microscopy. The optical and scanning electron microscope images of both samples are shown in Fig. 1, 2 for sections in both transversal and longitudinal directions. These rods have an average grain size of $50 \mu \mathrm{m}$ and $40 \mu \mathrm{m}$, respectively. The copper and silver phases present in the grains in sample $d_{1}$ and $d_{2}$ exhibit a crystallographic texture. The crystallographic texture is measured using the bulk $\mathrm{X}$ ray diffraction (XRD) method. The resulting pole figures of sample $d_{1}$ and $d_{2}$ in Fig. 3 (top 

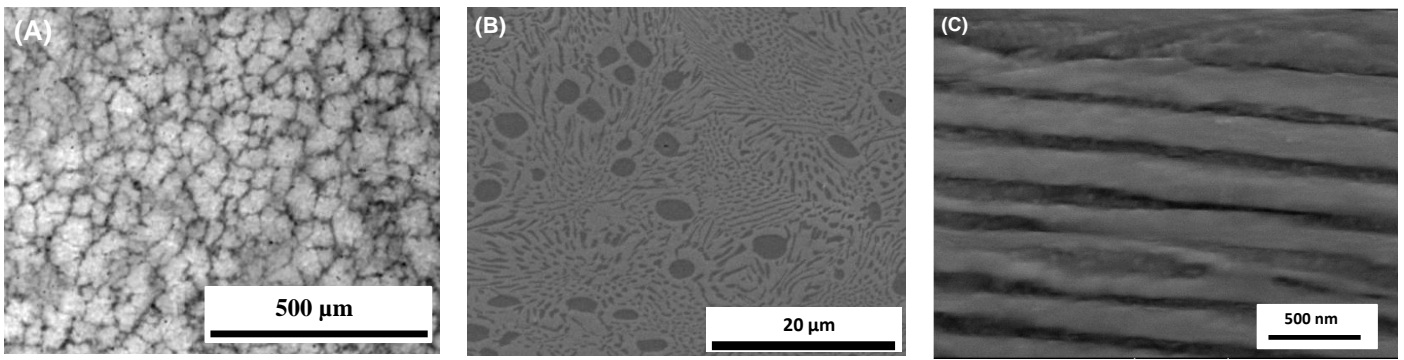

Figure 1: Microstructure of $\mathrm{Cu}-\mathrm{Ag}$ drawn material $d_{1}$ : (A), (B) grain structure and lamellae arrangement in transverse direction, $(\mathrm{C})$ lamellar structure in longitudinal direction
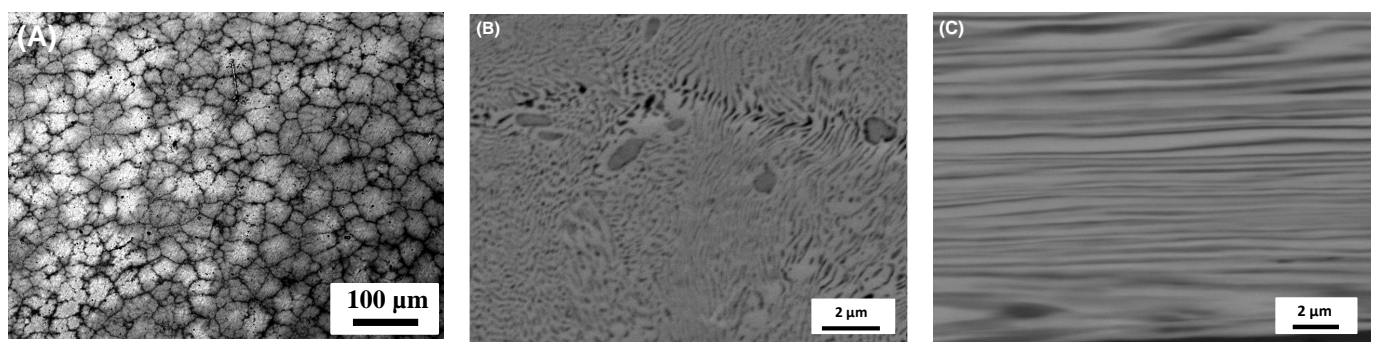

Figure 2: Microstructure of $\mathrm{Cu}-\mathrm{Ag}$ drawn material $d_{2}$ : (A), (B) grain structure and lamellae arrangement in transverse direction, $(\mathrm{C})$ lamellar structure in longitudinal direction
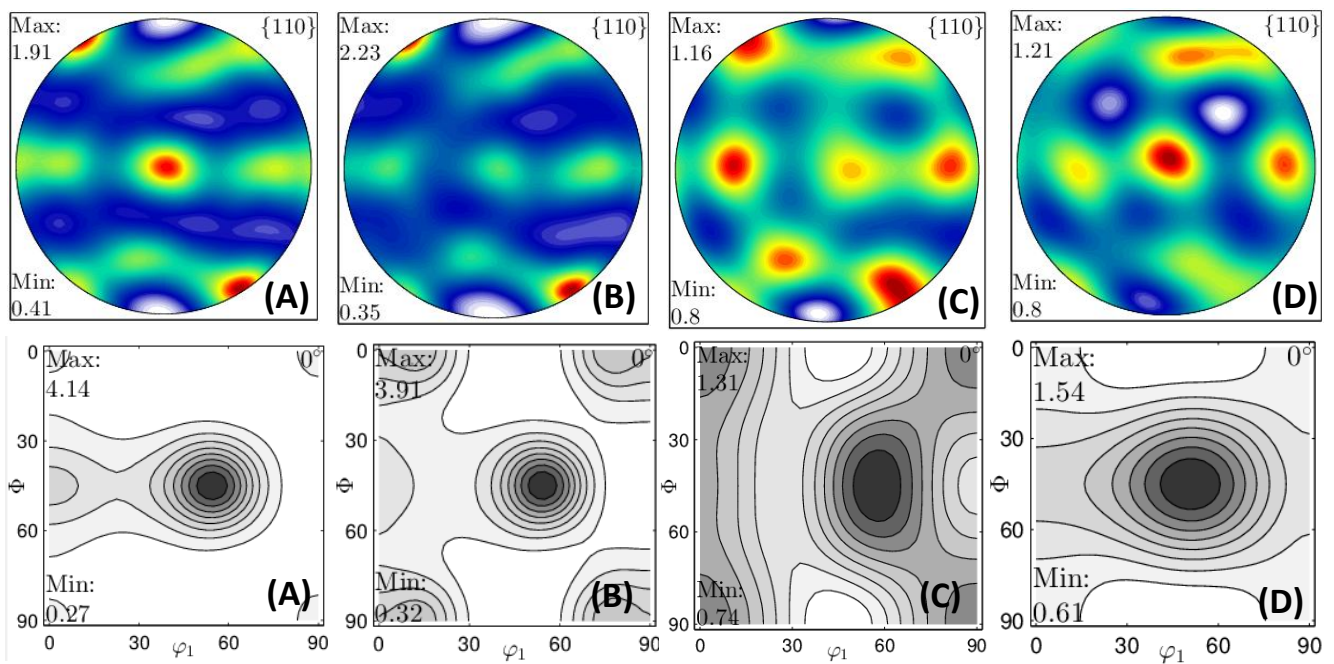

Figure 3: Sample $d_{1}$ : pole figures $\{110\}$ (top figure), (A) measured $\mathrm{Cu}$ phase, (B) measured $\mathrm{Ag}$ phase, (C) $\mathrm{Ag}$ phase approximated by 100 orientations, and (D) Cu phase approximated by 100 orientations. ODF (bottom figure) sections of $\varphi_{2}=0^{\circ}$ in the space of Euler angles, (A) measured Cu phase, (B) measured Ag phase, (C) Ag phase approximated by 100 orientations, and (D) Cu phase approximated by 100 orientations. 

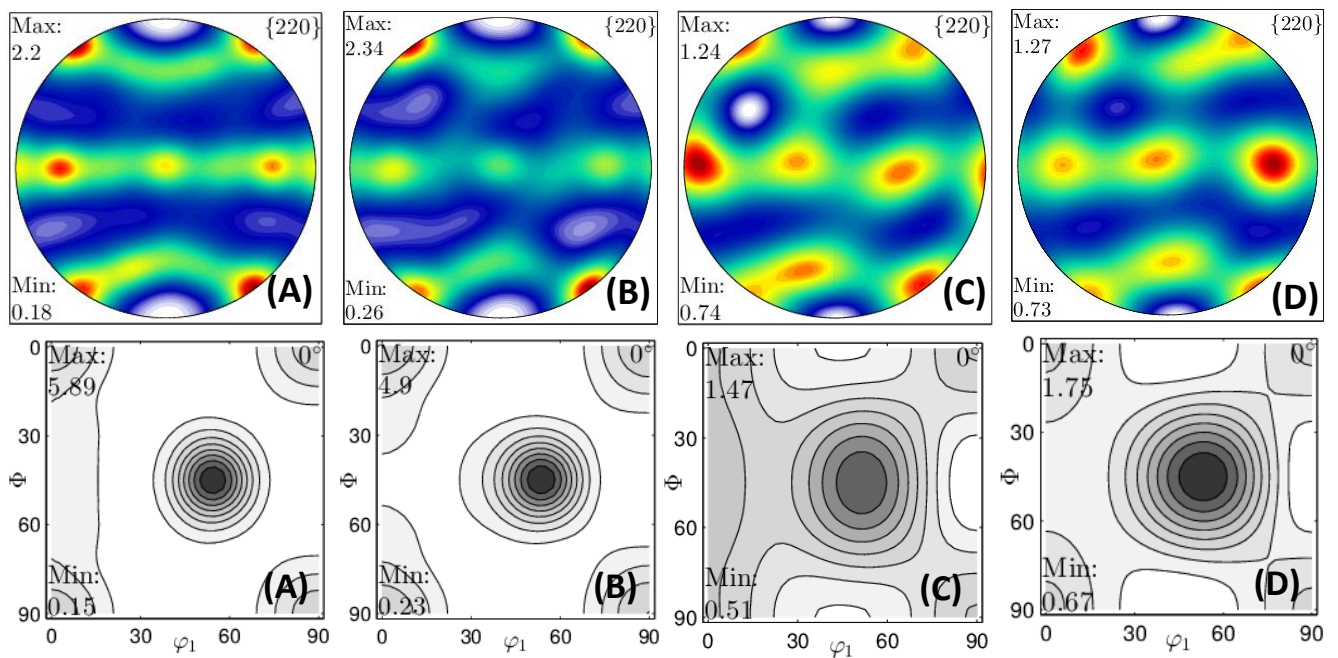

Figure 4: Sample $d_{2}$ : pole figures $\{220\}$ (top figure), (A) measured $\mathrm{Cu}$ phase, (B) measured Ag phase, and (C) $\mathrm{Cu}$ phase approximated by 100 orientations and (D) Ag phase approximated by 100 oreintations. ODF (bottom figure) sections of $\varphi_{2}=0^{\circ}$ in the space of Euler angles, (A) measured Cu phase, (B) measured Ag phase and (C) $\mathrm{Cu}$ phase approximated by 100 orientations, and (D) Ag phase approximated by 100 oreintations.

figure), Fig. 4 (top figure) exhibit a strong texture. Besides, the ODFs of both the samples are presented in the space of Euler angles (see Fig. 3 (bottom figure), Fig. 4 (bottom figure)). The matlab toolbox MTEX [22], [3], [23], [4] is used to reconstruct the orientation distribution function (ODF) from the XRD pole figures with the crystal orientation densities in a 3D orientation space defined by the three Euler angles $\phi_{1}, \varphi$ and $\phi_{2}$. These pole figures and ODFs are approximated by the MTEX algorithm by a set of 100 discrete orientations (see Fig. 3 C, D and Fig. 4 C, D). These 100 grain orientations should be representative enough to predict the mechanical behavior and are a good compromise between the accuracy of the results and the computational time. The mechanical behavior and texture have been validated by the experimental data.

\subsection{Macroscale compression tests}

The compression tests are performed for both cylindrical samples in the two directions (longitudinal and transversal) on a universal testing machine. These tests have been performed at room temperature for a constant strain rate of $10^{-4} s^{-1}$. The experimental results are reported in terms of true stress and true strain (see Fig. 7, 8). More details about the compression tests and microstructural observations are given in Dodla et al. [14].

\section{Material model}

An elasto-viscoplastic single crystal constitutive model is introduced in the lamellar structure of the $\mathrm{Cu}$ and $\mathrm{Ag}$ phases. The model is based on the concept of isomorphic elastic ranges [7]. The evolution of the plastic deformation is accounted for by means of a second-order tensor, called plastic transformation $\mathbf{P}$. The tensor $\mathbf{P}$ relates the variables in the undistorted placement to the reference placement. In the following equations, tilde $(\sim)$ refers to all variables which are described with respect to the initially undistorted lattice base.

\subsection{Elastic law}

For a prescribed deformation in terms of Green's strain tensor $\tilde{\mathbf{E}}^{G}$, the second Piola-Kirchhoff stress tensor $\tilde{\mathbf{T}}^{2 P K}$ is given by an anisotropic Hooke's law

$$
\mathbf{T}^{2 P K}=\mathbf{k}_{p}(\mathbf{C})=\mathbf{P k}_{0}\left(\mathbf{P}^{T} \mathbf{C P}\right) \mathbf{P}^{T}
$$


with

$$
\tilde{\mathbf{E}}^{G}=\frac{1}{2}(\tilde{\mathbf{C}}-\mathbf{I}), \quad \tilde{\mathbf{C}}=\tilde{\mathbf{F}}^{T} \tilde{\mathbf{F}}
$$

In Equation 1, the current elastic law $\mathbf{k}_{p}$ has been represented by a constant reference elastic law $\mathbf{k}_{0}$ and a plastic transformation $\mathbf{P}[7],[10]$. Equation 2 is the time-independent reference elastic law. Here $\mathbf{F}$ is the deformation gradient and $\tilde{\mathbf{F}}(:=\mathbf{F P})$ is the elastic part of deformation gradient describing elastic stretch and rigid rotation. $\tilde{\mathbb{K}}$ denotes the fourth-order constant stiffness tetrad for a cubic crystal. The tilde indicates that the $\tilde{\mathbb{K}}$ is formulated with respect to the undistorted placement. The stiffness tetrad $\mathbb{K}$ is represented by the six by six matrices and the components refer to the normalised orthonormal basis $\mathbf{B}_{\alpha}$ of symmetric second-order tensors [12, 9], i.e., $K_{\alpha \beta}=\mathbf{B}_{\alpha}: \mathbb{K}\left[\mathbf{B}_{\beta}\right]$

$$
\begin{aligned}
& \mathbb{K}=\left[\begin{array}{cccccc}
K_{11} & K_{12} & K_{12} & 0 & 0 & 0 \\
& K_{11} & K_{12} & 0 & 0 & 0 \\
& & K_{11} & 0 & 0 & 0 \\
& & & 2 K_{44} & 0 & 0 \\
& \text { sym. } & & & 2 K_{44} & 0 \\
& & & & & 2 K_{44}
\end{array}\right] \mathbf{B}_{\alpha} \otimes \mathbf{B}_{\beta} \\
& \mathbf{B}_{1}=\mathbf{e}_{1} \otimes \mathbf{e}_{1}, \mathbf{B}_{2}=\mathbf{e}_{2} \otimes \mathbf{e}_{2}, \mathbf{B}_{3}=\mathbf{e}_{3} \otimes \mathbf{e}_{3} \\
& \mathbf{B}_{4}=\frac{\sqrt{2}}{2}\left(\mathbf{e}_{2} \otimes \mathbf{e}_{3}+\mathbf{e}_{3} \otimes \mathbf{e}_{2}\right) \\
& \mathbf{B}_{5}=\frac{\sqrt{2}}{2}\left(\mathbf{e}_{1} \otimes \mathbf{e}_{3}+\mathbf{e}_{3} \otimes \mathbf{e}_{1}\right) \\
& \mathbf{B}_{6}=\frac{\sqrt{2}}{2}\left(\mathbf{e}_{1} \otimes \mathbf{e}_{2}+\mathbf{e}_{2} \otimes \mathbf{e}_{1}\right)
\end{aligned}
$$

Copper and silver materials have a face centered cubic (fcc) crystal structure. The three independent elastic constants for copper are $K_{11}=170 \mathrm{GPa}, K_{12}=124 \mathrm{GPa}$ and $K_{44}=75 \mathrm{GPa}$, and for silver $K_{11}=123.99 \mathrm{GPa}, K_{12}=93.67 \mathrm{GPa}$ and $K_{44}=46.12 \mathrm{GPa}[29],[27]$.

\subsection{Flow rule}

In general, the fcc materials exhibit crystallographic slip in $\{111\}\langle 110\rangle$ slip systems. These 12 slip systems are described by the Schmid tensors $\tilde{\mathbf{S}}^{\alpha}:=\tilde{\mathbf{d}}_{\alpha} \otimes \tilde{\mathbf{n}}^{\alpha}$, which are given by the slip direction $\tilde{\mathbf{d}}_{\alpha}$ and the slip plane normal $\tilde{\mathbf{n}}^{\alpha}$. The resolved shear stress $\tau^{\alpha}$ in a slip system $\alpha$ can be calculated as

$$
\tau^{\alpha}:=\tilde{\mathbf{C}} \tilde{\mathbf{T}}^{2 P K}: \mathbf{S}^{\alpha}
$$

An evolution of the plastic transformation $\mathbf{P}$ is given in terms of the shear rate $\dot{\gamma}_{\alpha}$ and Schmid tensor $\tilde{\mathbf{S}}^{\alpha}$

$$
\mathbf{P}^{-1} \dot{\mathbf{P}}=-\sum_{\alpha} \dot{\gamma}^{\alpha} \tilde{\mathbf{S}}^{\alpha}
$$

The kinetics of dislocation motion have been elaborated by the relationships between the resolved shear stress and the plastic shear rate $\dot{\gamma}^{\alpha}$ of the slip system $\alpha$ by using the power law [26]

$$
\dot{\gamma}^{\alpha}=\dot{\gamma}_{0} \operatorname{sgn}\left(\tau^{\alpha}\right)\left|\frac{\tau^{\alpha}}{\tau_{c}(\gamma)}\right|^{m}
$$




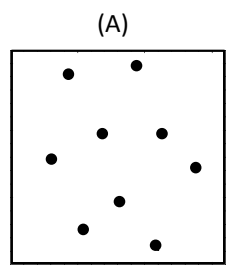

(B)

(C)
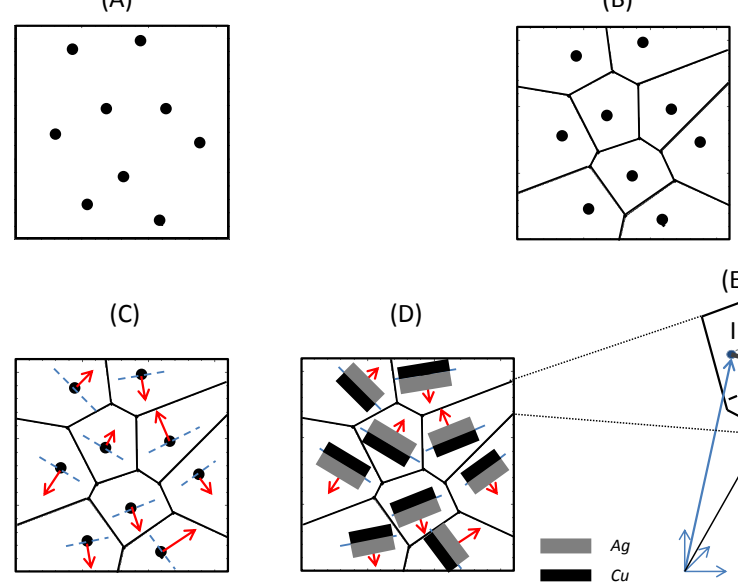

(D)

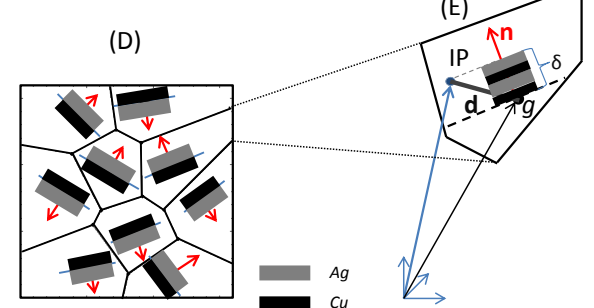

Figure 5: (A) Two dimensional view of Voronoi points, (B) Fixed boundary Voronoi points (black dots), (C) interface normal $\mathbf{n}$ for each Voronoi point, (D), (E) generation of lamellar structure $\mathrm{Cu}, \mathrm{Ag}$ along the interface normal $\mathbf{n}$ inside the grain $g$.

where $\dot{\gamma}_{0}$ is a constant reference shear rate, and the exponent $m$ measures the strain sensitivity of the material. The initial conditions of the evolution equation $(9)$ are $\tilde{\mathbf{F}}(t=0)=\mathbf{Q}(t=0) \in$ $S O(3)$. The orientation of the crystal is given by a proper orthogonal tensor $\mathbf{Q}(t):=\mathbf{g}_{i}(t) \otimes \mathbf{e}_{i}$. Here $\mathbf{e}_{i}$ is the orthonormal base vectors of a fixed Cartesian coordinate system and $\mathbf{g}_{i}$ is an orthonormal lattice vector. The initial resolved shear stress at time $t=0$ is given as $\tau_{c}(0)=\tau_{c_{0}}$.

\subsection{Hardening rule}

A simple, most popular ansatz for the two types of hardening (self, latent) is the linear hardening rule [25],[7].

$$
{\dot{\tau_{c}}}^{\alpha}=\sum_{\beta} h_{\alpha \beta} \dot{\gamma}^{\beta}, \quad h_{\alpha \beta}=q_{\alpha \beta} \theta(\gamma)
$$

where $\theta(\gamma)=\frac{d \tau_{c}^{\alpha}}{d \gamma}$ and $q_{\alpha \beta}$ are the matrix components which account for self and latent hardening of the crystal. For the fcc cubic crystal having $12\{111\}\langle 110\rangle$ slip systems, we consider $q_{\alpha \beta}$ equal to 1.0 for the coplanar slip systems and equal to 0.9 for noncoplanar systems. The evolution of the critical resolved shear stress of all slip systems as a function of shear $\gamma$ is described by a Voce-type hardening law [43]

$$
\tau_{c}^{\alpha}(\gamma)=\tau_{c_{0}}+\left(\tau_{s}+\theta_{\infty} \gamma\right)\left(1-\exp \left(-\theta_{0} \gamma / \tau_{s}\right)\right)
$$

with

$$
\gamma=\int \sum_{\alpha}\left|\dot{\gamma}_{\alpha}\right| \mathrm{dt}
$$

$\gamma$ is given by the sum of shear rates of all slip systems. The Voce type hardening rule consists of four hardening paramaters, namely the initial resolved shear stress $\tau_{c_{0}}$, a saturation stress $\tau_{s}$, an initial hardening modulus $\theta_{0}$, and a remaining hardening modulus $\theta_{\infty}$. The material model has been implemented into the user subroutine (UMAT) of the finite element package (ABAQUS) [1]. A Newton-Raphson iteration has been done using a backward Euler scheme [11]. 


\section{Finite Element modeling of the Lamellar Structure}

\subsection{Three dimensional Poisson-Voronoi tessellation}

Most of the real microstructures are complex in nature. A reasonable assumption for the topological approximation of real materials is a three-dimensional Poisson-Voronoi tessellation. From material science and metallurgy, the Voronoi tessellation points can be understood as resulting from the crystal growth process in all directions [33, 38]. In this work, the grain microstructure is given as Voronoi tessellations in three dimensions. The Voronoi points of the grains are created by a Poisson process, so that these points are distributed uniformly (Poisson distribution). The number of grains represent the number of Voronoi points. The periodic microstructure is generated by copying the Voronoi points in all the directions. Therefore, a total of 27 cubes for the periodic cell in the 3D space has been produced. A center cube in the above created Voronoi structure is taken as the representative volume element (RVE). For each Voronoi point $g$, the interface normal is generated randomly, i.e., for every grain there is an interface normal vector $\mathbf{n}$ which provides the information of the lamellar direction. Besides, the same interface normal is generated for the incomplete grains at opposite boundaries since the microstructure is periodic. Voronoi points are mapped to the lamellar structure of alternating layers of $\mathrm{Cu}$ and $\mathrm{Ag}$ (see Fig. 5). The distance vector $\mathbf{d}$ is calculated between the integration point IP and the Voronoi point $\mathrm{g}$. This vector $\mathbf{d}$ is projected on the interface normal vector to calculate the thickness $\delta=\mathbf{d} \cdot \mathbf{n}$ of the lamellae (Fig. 5 E). From the micrographs it is clear that the microstructure consists of alternate layers of $\mathrm{Cu}$ and Ag lamella. Therefore, the thickness $\delta\left(=\delta_{C u_{1}}+\delta_{A g_{1}}+\delta_{C u_{2}}+\delta_{A g_{2}}, ..\right)$ value is used for the construction of the lamellar structure (see Fig. $5 \mathrm{E}$ ). The lamella thickness of each phase considered in the FE simulations is simply the volume fraction $(v f)$ of the polycrystals, i.e., $v f_{C u}=\delta_{C u}=0.37$ and $v f_{A g}=\delta_{A g}=0.63$. The crystal orientation is assigned to the lamellar phases based on the information for a given integration point whether it belongs to the $\mathrm{Cu}$ phase or the $\mathrm{Ag}$ phase and to which grain. This information can be obtained from the user material subroutine UMAT in ABAQUS. Each lamella $(\mathrm{Cu}, \mathrm{Ag})$ inside the grain has the same initial crystallographic orientation with respect to the grain [14]. In the experimental investigations [14], few shear bands or twinned regions and also few proeutectic regions are observed in the experiments. These effects are not considered in the model. The orientation of each lamella $(\mathrm{Cu}, \mathrm{Ag})$ varies from one grain to another depending on the initial texture of the sample $\left(d_{1}, d_{2}\right)$. In general, the $\mathrm{Cu} / \mathrm{Ag}$ lamellar structure and grains are in different length scales as shown in the real microstructures $d_{1}, d_{2}$. However, the present model is able to represent the combined effect of lamellar structure and grain structure. Hence the finite element simulations are able to solve the lamellar two phase $\mathrm{Cu}-\mathrm{Ag}$ polycrystals.

\subsection{Finite Element Mesh and Periodic boundary conditions}

Finite element calculations have been performed by the finite element software (ABAQUS). In the RVE simulations, a three dimensional eight-noded linear element (C3D8) is chosen. The Gauss points for each grain can be determined based on the minimum distance between the grain and Gauss point. For the application of the periodic boundary conditions three artificial nodes are considered. Each artificial node has a three displacement degrees of freedom. In total, nine additional displacement degrees of freedom have been assigned to the components of the average displacement gradient $\overline{\mathbf{H}}$. All boundary nodes (corner, surface and edge) are formed into pairs $\mathbf{x}_{0}^{+}$ and $\mathbf{x}_{0}^{-}$, where the surface normal vector is equilibrated on the opposite boundaries

$$
\mathbf{n}_{0}^{+}=-\mathbf{n}_{0}^{-} .
$$

For given pairs of $\mathbf{x}_{0}^{+}$and $\mathbf{x}_{0}^{-}$with Equation 13, the formulation of periodic boundary conditions in terms of an imposed average displacement gradient $\overline{\mathbf{H}}$ and stress $\mathbf{T}$ can be expressed as

$$
\mathbf{u}^{+}-\mathbf{u}^{-}=\overline{\mathbf{H}}\left(\mathbf{x}_{0}^{+}-\mathbf{x}_{0}^{-}\right), \quad \mathbf{t}^{+}=-\mathbf{t}^{-}
$$

Here $\mathbf{t}^{ \pm}$are the traction vectors on the surfaces. The stress field is equilibrated on the opposite boundaries (see also [17]). The periodic boundary conditions are implemented into the three artificial nodes in the RVE using the python script by the above mentioned equation (Equation $14)$. 


\subsection{Homogenization of stresses}

Prescribing the displacement gradient $\overline{\mathbf{H}}$, the stresses have been computed based on the assumption of equivalence of work on the micro and mesoscale [24], the $1^{\text {st }}$ Piola-Kirchhoff stresses on the local $\left(\mathbf{T}^{1 P K}\right)$ and the average $\left(\overline{\mathbf{T}}^{1 P K}\right)$ stresses are related by

$$
\overline{\mathbf{T}}^{1 P K}=\frac{1}{V} \int_{V} \mathbf{T}^{1 P K} d V
$$

The reference volume $V$ in Equation 15 is taken as the volume average of the RVE. By homogenization over the RVE, the reaction force vectors and displacements are extracted from three artificial nodes. Hence, corresponding stresses and strains are calculated using the constitutive model given in Section 3.

\subsection{Mesh convergence}

A convergence study has been carried out for both polycrystals $d_{1}, d_{2}$. We used the h-method of convergence, i.e., the number of elements and hence of the nodes on each side with variable size $h$ has been increased in order to achieve convergence. The number of elements of each side of the RVE has been studied in steps of 16, 32, 40 and 48 elements. We concluded that a sufficient mesh size on each side of RVE is 40 elements, which has been applied to all the finite element simulations.
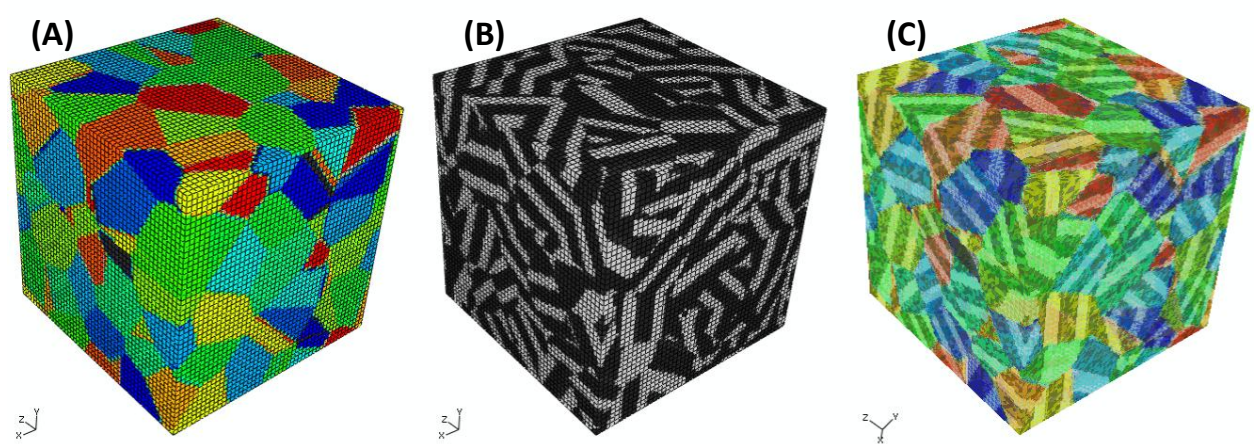

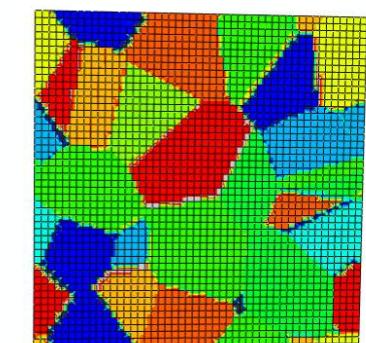

(A)

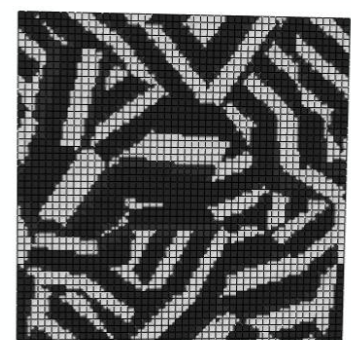

(B)

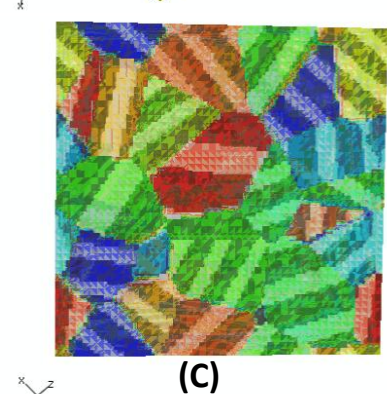

$y^{2}$

(C)

Figure 6: Top figure: (A) three dimensional view of periodic Voronoi microstructure, (B) $\mathrm{Cu}$ and Ag lamellar structure inside the grains, and $(\mathrm{C})$ combination of both grain structure and lamellar structure of $\mathrm{Cu}, \mathrm{Ag}$ phases inside the RVE, Bottom figure: (A) plane view of periodic Voronoi microstructure, (B) plane view of $\mathrm{Cu}$ and $\mathrm{Ag}$ lamellar structure inside the grains, and $(\mathrm{C})$ combination of grain and lamellar structure

\subsection{Identification of material parameters of the sample with $d_{1}$ and $d_{2}$}

For the model used, two viscous flow parameters $\left(\dot{\gamma}_{0}, m\right)$ in the viscoplastic power law (see Equation 10) are needed. The initial shear rate $\dot{\gamma}_{0}$ can be selected arbitrarily, and we consider $10^{-4} s^{-1}$ for both the phases for the sample $d_{1}$ and $d_{2}$, and the strain rate sensitivity parameter $m=80$ is taken from the literature [29]. We used the Taylor model for the identification of the Voce hardening parameters for the two polycrystals $\left(d_{1}, d_{2}\right)$. We begin with the Voce hardening 

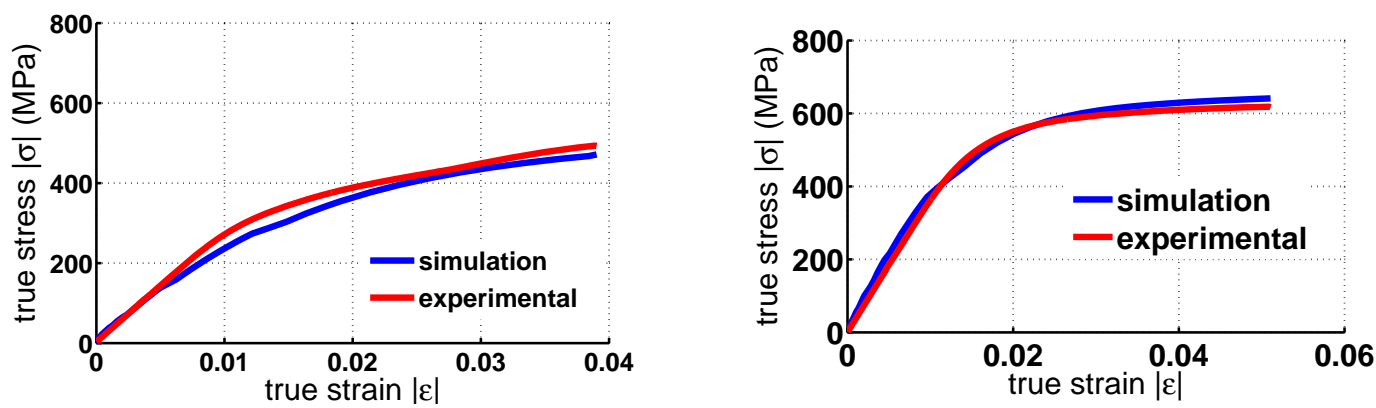

Figure 7: Prediction and measured true stress-strain curves for the compression test of sample $d_{1}$ (left figure) and sample $d_{2}$ (right figure)

parameters given in [8] for both the $\mathrm{Cu}$ and $\mathrm{Ag}$ phases. The constitutive equations are similar to those given in the material model (Section 3). The hardening parameters for both the samples are identified by simulating a uniaxial compression test in longitudinal direction and comparing the results with the measured experimental data [14]. The minimization problem has been solved using the minpack package [37], in which the Levenberg-Marquardt algorithm has been applied. The finite element simulations have been carried out at room temperature for a constant strain rate of $10^{-4} s^{-1}$ upto a compressive deformation of $4 \%$ for the sample $d_{1}$ (see Fig. 7 (left figure)) and $5 \%$ for the sample $d_{2}$ (see Fig. 7 (right figure)). The numerically predicted stress-strain data is similar to the experimental data obtained from compression experiments of both samples $d_{1}$, $d_{2}$. All identified hardening parameters and flow parameters used in the FE simulation are listed in Table 1.

\section{Validation results of samples $d_{1}$ and $d_{2}$ in transverse direction}

\subsection{Stress-strain behavior}

Three dimensional finite element simulations of compression tests have been employed to validate the defined model in transverse $(Z)$ direction for both the samples. From these tests, the true stress-strain behavior (see Fig. 8) can be obtained, and this curve is considered to be a validation of hardening parameters and initial texture. The comparison of the simulated stress-strain curves with the experimentally obtained stress-strain curves will reveal the validity of the materal model. As one can see in Fig. 8 (left), numerical simulations are in good agreement with the experimental measurements of the drawn sample $d_{1}$. However, there is a slight deviation at strains below $3 \%$ of the drawn sample $d_{2}$ as shown in Fig. 8 (right). The deviation may be due to heterogenity in the microstructure. It is observed that the microstructure of sample $d_{2}$ shows a reduction in grain size from the center to the edge of the rod, because the rod is more compressed at the outer surface during the drawing operation. Moreover, the approximation by only 100 grains is rather small, although sufficient to capture the main features of the real texture.

Table 1: Material parameters

\begin{tabular}{cccllll}
\hline Material & $\dot{\gamma}_{0}\left[\mathrm{~s}^{-1}\right]$ & $m[-]$ & $\tau_{c_{0}}[M P a]$ & $\tau_{s}[M P a]$ & $\theta_{0}[M P a]$ & $\theta_{\infty}[M P a]$ \\
\hline sample: $d_{1}$ & & & & & & \\
$\mathrm{Cu}$ & 0.0001 & 80 & 5.5 & 167.6 & 7964 & 9.2 \\
$\mathrm{Ag}$ & 0.0001 & 80 & 5 & 200.9 & 4501 & 20 \\
\hline sample: $d_{2}$ & & & & & & \\
$\mathrm{Cu}$ & 0.0001 & 80 & 5.5 & 241.4 & 14000 & 105 \\
$\mathrm{Ag}$ & 0.0001 & 80 & 5 & 227.6 & 12746 & 50 \\
\hline
\end{tabular}



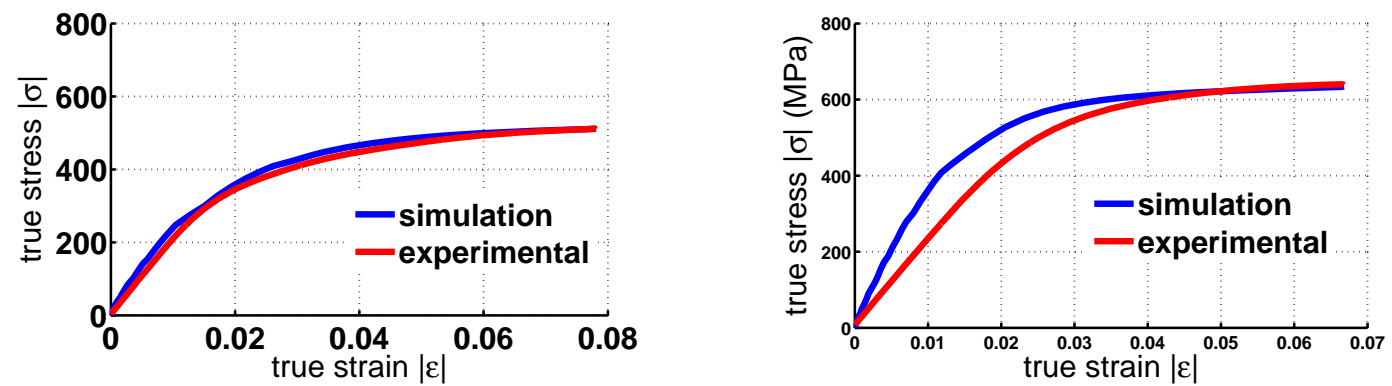

Figure 8: Measured and validated stress-strain curves for the compression test of sample $\left(d_{1}\right)$ (left figure) and sample $d_{2}$ (right figure)
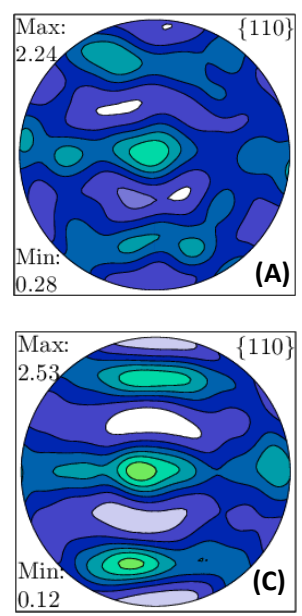
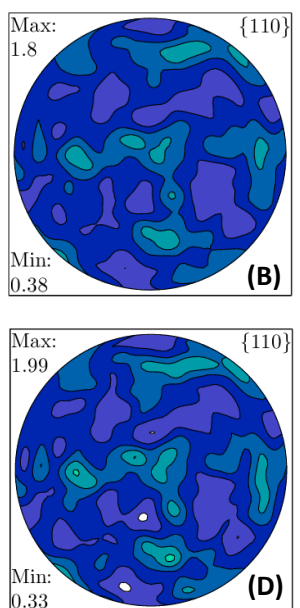
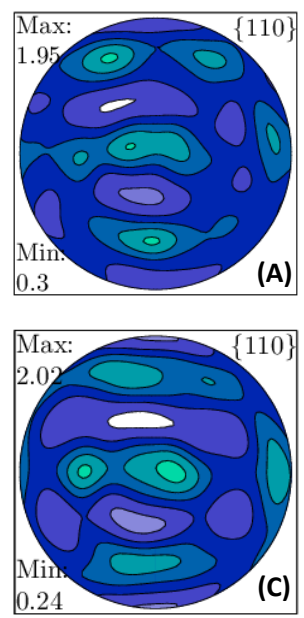
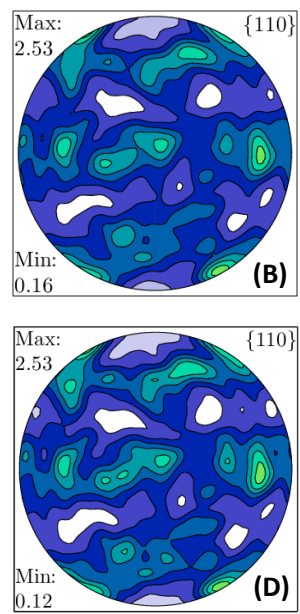

Figure 9: Pole figures $d_{1}$ : (A), (B) measured and sim- Figure 10: Pole figures $d_{2}$ : (A), (B) measured and simulated $\mathrm{Cu}$ phase, (C), (D) measured and simualted $\mathrm{Ag}$ ulated $\mathrm{Cu}$ phase, (C), (D) measured and simualted $\mathrm{Ag}$ phase phase

\subsection{Crystallographic texture}

The measured and simulated textures in this article are presented in terms of (110) pole figures and $\phi_{2}\left(=0^{\circ}, 45^{\circ}, 65^{\circ}\right)$ sections of the orientation distribution function (ODF) in the space of Euler angles $\left(\phi_{1}, \varphi\right) \leq 90^{\circ}$. The final texture of both samples $\left(d_{1}, d_{2}\right)$ after compression testing is presented as the pole figures and ODF shown in Figures 9, 10, 11 and 12. The important observations of the measured textures of sample $d_{1}$ and $d_{2}$ are as taken as follows. (1) Both $\mathrm{Cu}$ and Ag phases develop the same alloy type texture. (2) The texture components observed in the $\mathrm{Ag}$ and $\mathrm{Cu}$ phase have a strong brass component. In the ODF plot (see Fig. 11 and 12), the $\phi_{2}$ sections at $0^{\circ}$ and $45^{\circ}$ expose a strong brass component. However, some weak texture components are observed in the finite element model calculations for the $\phi_{2}$ section at $65^{\circ}$. This effect is a general characteristic for texture simulations by a small number of grains. (3) Both phases have the same texture features with small differences in the intensities of the texture, and are similar to those reported previously for the $\mathrm{Cu}-24 \mathrm{wt} \% \mathrm{Ag}$ rolled sheets [13]. Besides the numerical predictions with the approximated discrete 100 orientations of both the samples capture the above mentioned experimental observations of the crystallographic texture. However, the texture is overpredicted in both the samples. The reason for this is that the number of orientations is rather small to represent the complete texture. Nevertheless, the model describes all the important texture components of the aforementioned experimental measurements. For predicting the textures of two polycrystals more precisely under loading, many more orientations must be incorporated in the model. In that case, however, the finite element simulations would become prohibitively expensive for the complex microstructures. 

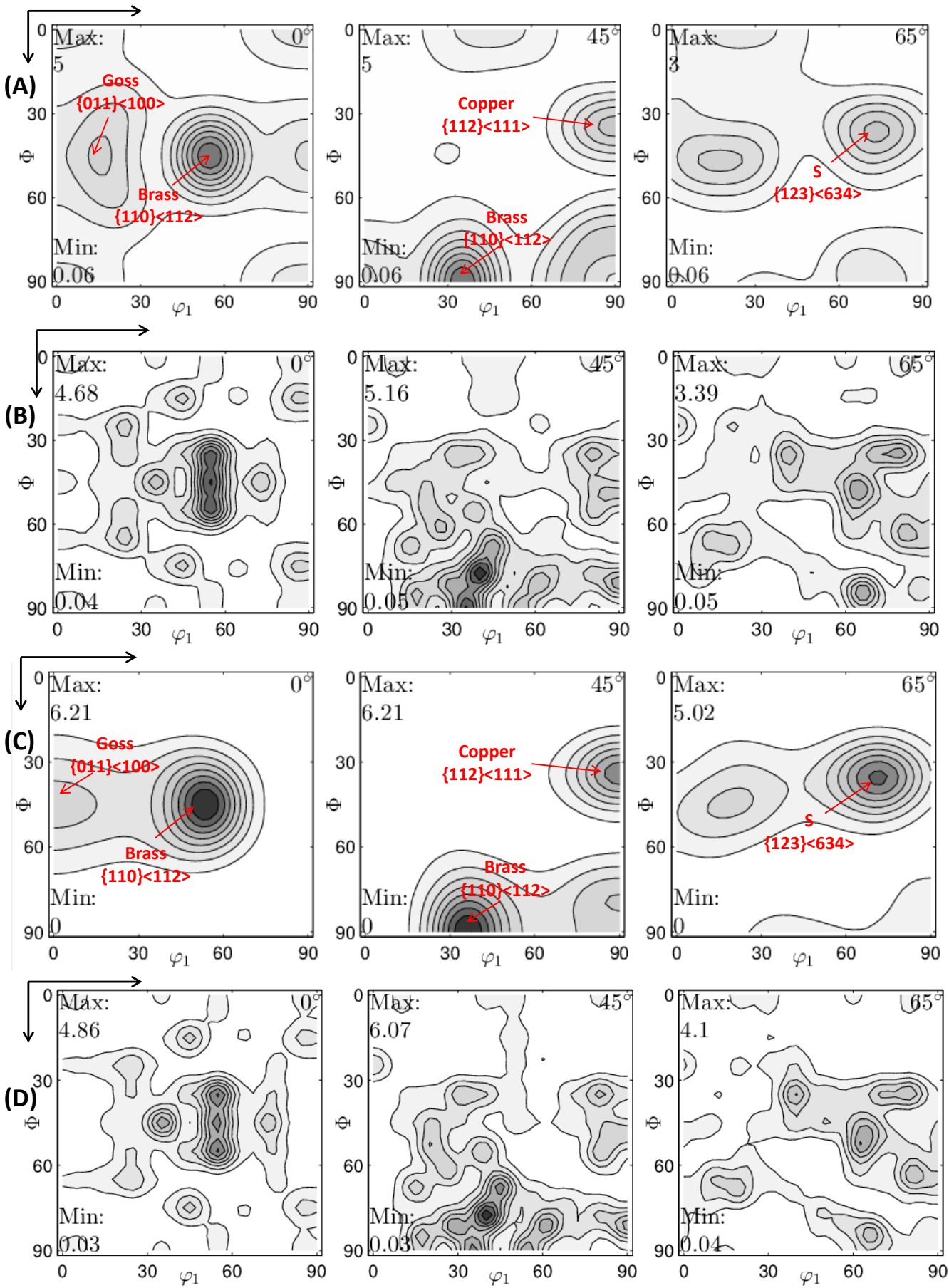

Figure 11: Sample $d_{1}$ : ODF (A) measured $\mathrm{Cu}$ phase, (B) simulated $\mathrm{Cu}$ phase (C) measured Ag phase and (D) simulated Ag phase.

\section{Summary and conclusions}

In this paper, two different cold drawn textured polycrystals $\left(d_{1}, d_{2}\right)$ are numerically investigated. The lamellar nature of the $\mathrm{Cu}$ and $\mathrm{Ag}$ phases are studied at the lamella scale by performing 3D finite element simulations. Besides, simulations of the texture evolution of two polycrystals have been validated by the experimentally measured texture. In the finite element simulation, 

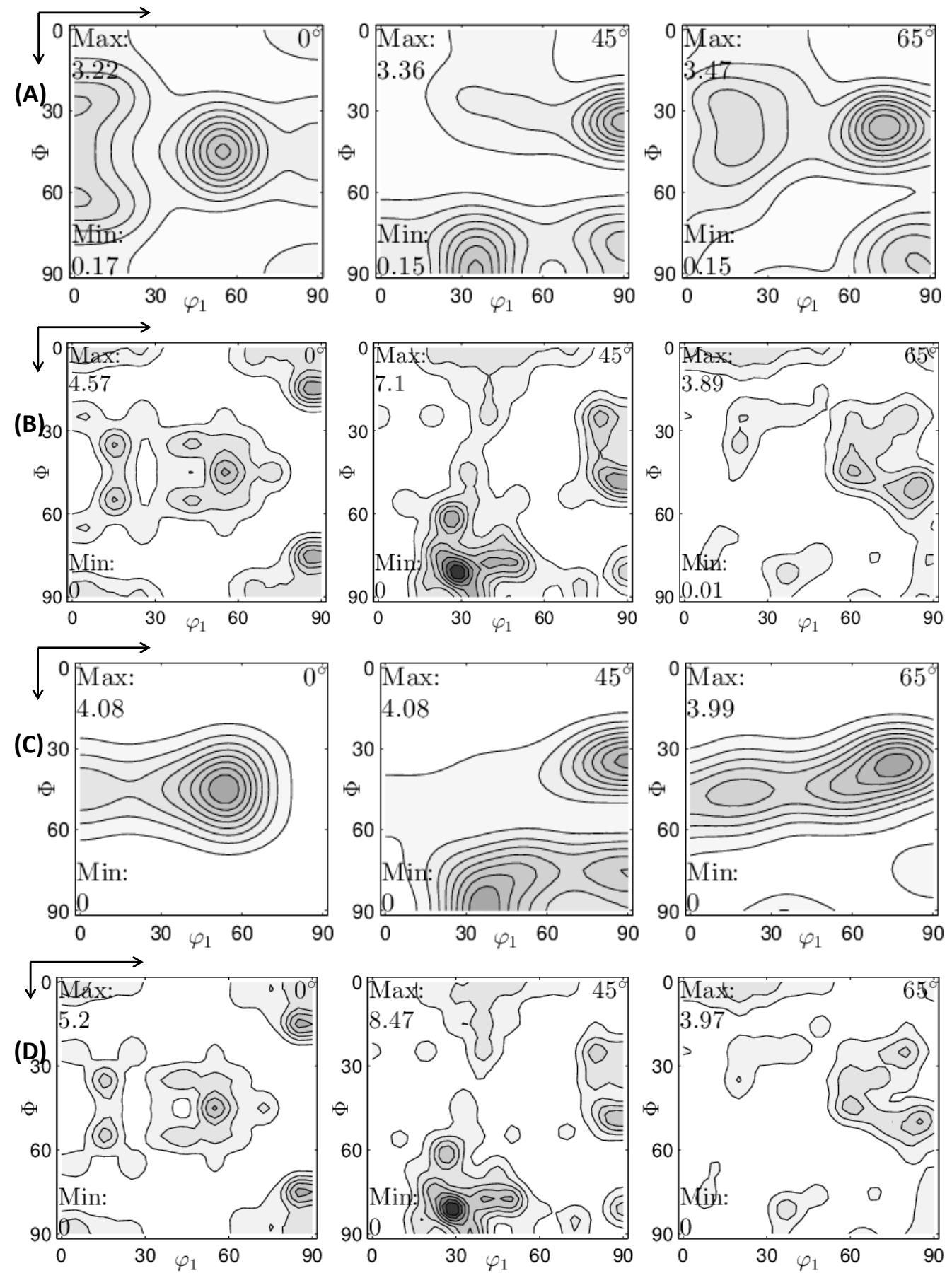

Figure 12: Sample $d_{2}$ : ODF (A) measured $\mathrm{Cu}$ phase, (B) simulated $\mathrm{Cu}$ phase (C) measured Ag phase and (D) simulated Ag phase.

the microstructure is represented as a periodic Voronoi tessellation. The finite element simulation (RVE) captures the two phase lamellar morphology inside the grains. The compression tests have been performed in the transverse direction ( $\mathrm{Z}$ direction) to validate the material parameters of the aforementioned two phase lamellar $\mathrm{Cu}$-Ag polycrystals. From the numerical investigations of these two polycrystals $\left(d_{1}, d_{2}\right)$, we obtain the following conclusions.

1. The material parameters of two phase textured polycrystals using the crystal plasticty ma- 
terial model have been identified by means of macroscale compression tests (see Fig. 7 (left) and 7 (right)).

2. Using the crystal plasticity material model, the mechanical behavior of a two phase polycrystal $d_{1}$ is validated by the macroscopic experimental results, and the numerical model approximated by a 100 grains initial texture is well captured (see Fig. 8 (left)). In the case of the polycrystal $d_{2}$, the stress-strain behavior shows a small deviation at strains $<3 \%$ but the simulated stress-strain curve is in good accordance with the experimental curve at strains above $3 \%$ (see Fig. 8 (right)). Nevertheless, the deviation is in an acceptable range.

3. The numerical simulations reproduce the experimentally observed textures in both $\mathrm{Cu}-\mathrm{Ag}$ polycrystals $\left(d_{1}, d_{2}\right)$. Figure 9 to 12 reveals the simulated and measured textures for the $\mathrm{Cu}$ and $\mathrm{Ag}$ phases of sample $d_{1}$ and $d_{2}$. Both phases developed the same type of texture. The brass type texture component is developed in the $\mathrm{Cu}$ and $\mathrm{Ag}$ phases. The simulated textures are overpredicted with the measured texture results, but the important texture components (Goss, Brass, Copper and S) are well captured.

\section{Acknowledgements}

The financial support provided by the German Science Foundation (DFG) through GRK 1554 is acknowledged. We sincerely thank Thorsten Halle (Otto-von-Guericke University Magdeburg) for fruitful discussions. We thank H. Heyse and P. Thiem for performing the XRD measurements. We gratefully acknowledge valuable remarks from Jan Kalisch. Also, we would like to thank the editor and reviewers for their helpful comments and advices.

\section{References}

[1] ABAQUS, 1990. Reference Manuals, Hibbitt, Karlsson and Sorensen. Addison-Wesley, Inc., Providence, RI.

[2] Asano, T., Sakai, Y., Inoue, K., Oshikiri, M., Maeda, H., 1993. High-field pulsed Magnet Wound of Cu-Ag Alloy Wire. Japanese Journal of Applied Physics 32, L1027-L1029.

[3] Bachmann, F., Hielscher, R., Jupp, P. E., Pantleon, W., Schaeben, H., Wegert, E., Dec 2010. Inferential statistics of electron backscatter diffraction data from within individual crystalline grains. Journal of Applied Crystallography 43 (6), 1338-1355.

URL http://dx.doi.org/10.1107/S002188981003027X

[4] Bachmann, F., Hielscher, R., Schaeben, H., 2010. Texture analysis with MTEX-Free and Open Source Software Toolbox. Solid State Phenomena 160, 63-68.

URL http://dx.doi.org/10.4028/www.scientific.net/SSP.160.63

[5] Benghalem, A., Morris, D. G., 1993. Microstructure and mechanical properties of concentrated copper-niobium alloys prepared by mechanical alloying. Materials Science and Engineering 161, 255-266.

[6] Benghalem, A., Morris, D. G., 1997. Microstructure and strength of wire-drawn Cu-Ag filamentary composites. Acta Materialia 45, 397-406.

[7] Bertram, A., 2012. Elasticity and Plasticity of Large Deformations - an Introduction, Third Edition. Springer Verlag, Berlin.

[8] Beyerlein, I. J., Mara, N. A., Bhattacharyya, D., Alexander, D. J., Necker, C. T., 2011. Texture evolution via combined slip and deformation twinning in rolled silver-copper cast eutectic nanocomposite. Acta Materialia 27, 121-146.

[9] Böhlke, T., 2001. Crystallographic Texture Evolution and Elastic Anisotropy: Simulation, Modeling and Applications. Shaker Verlag, Aachen. 
[10] Böhlke, T., Bertram, A., 2001. The evolution of Hooke's law due to texture development in FCC polycrystals. International Journal of Solids and Structures 38, 9437-9459.

[11] Böhlke, T., Risy, G., Bertram, A., 2006. Finite element simulation of metal forming operations with texture based material models. Modelling and Simulation in Material Science and Engineering 14, 365-387.

[12] Cowin, S., 1989. Properties of the anisotropic elasticity tensor. Quarterly J. of Mechanics \& Appl. Maths 42, 249-266.

[13] Davy, C. A., Han, K., Kalu, P. N., Bole, S. T., 2008. Examinations of Cu-Ag Composite Conductors in Sheet Forms. IEEE Transactions on Applied Superconductivity 18, 560-563.

[14] Dodla, S., Thiem, P., Krüger, M., Bertram, A., 2014. Investigations of microstructure and texture of Copper-Silver Composites. submitted to Metallurgical and Materials Transactions A.

[15] Embury, J. D., Hirth, J. P., 1994. On dislcation storage and the mechanical response of fine scale microstructures. Acta Metall. Mater. 42, 2051-2056.

[16] Gaganov, A., Freudenberger, J., Grünberger, W., Schultz, L., 2004. Microstructural evolution and its effect on the mechanical properties of $\mathrm{Cu}$-Ag microcomposites. Z. Metallkd. 95, 425432.

[17] Glüge, R., Weber, M., Bertram, A., 2012. Comparison of spherical and cubical statistical volume elements with respect to convergence, anisotropy, and localization behavior. Computational Materials Science 63, 91-104.

[18] Grünberger, W., Heilmaier, M., Schultz, L., 2001. Development of high-strength and highconductivity conductor materials for pulsed high-field magnets at dresden. Physica B 45, 643-647.

[19] Han, K., Embury, J. D., Sims, J. R., Campbell, L. J., Schneider Muntau, H. J., Pantsyrnyi, V. I., Shikov, A., Nikulin, A., Vorobieva, A., 1999. The fabrication, properties and microstructure of $\mathrm{Cu}-\mathrm{Ag}$ and $\mathrm{Cu}-\mathrm{Nb}$ composite conductors. Materials Science and Engineering A 267, 99-114.

[20] Heringhaus, F., 1998. Quantitative Analysis of the Influence of the Microstructure on Strength, Resistivity, and Magnetoresistance of Eutectic Silver-Copper. Shaker Verlag, Berlin.

[21] Heringhaus, F., Raabe, D., 1996b. Recent Advances in the Manufacturing of Copper-Base Composites. Journal of Materials Processing Technology 59, 367-372.

[22] Hielscher, R., Schaeben, H., Dec 2008. A novel pole figure inversion method: specification of the MTEX algorithm. Journal of Applied Crystallography 41 (6), 1024-1037. URL http://dx.doi.org/10.1107/S0021889808030112

[23] Hielscher, R., Schaeben, H., Siemes, H., 2010. Orientation distribution within a single hematite crystal. Mathematical Geosciences 42, 359-375. URL http://dx.doi.org/10.1007/s11004-010-9271-z

[24] Hill, R., 1963. Elastic properties of reinforced solids: Some theoretical principles. Journal of the Mechanics and Physics of Solids 11, 357-372.

[25] Hill, R., 1966. Generalized constitutive relations for incremental deformation of metal crystals by multislip. Journal of the Mechanics and Physics of Solids 14, 95-102.

[26] Hutchinson, J., 1976. Bounds and self-consistent estimates for creep of polycrystalline materials. Proc. R. Soc. London A 348, 101-127. 
[27] Jia, N., Raabe, D., Zhao, X., 2014. Texture and microstructue evolution during noncrystallographic shear banding in a plane strain compressed $\mathrm{Cu}-\mathrm{Ag}$ metal matrix composite. Acta Materialia 76, 238-251.

[28] Jia, N., Roters, F., Eisenlohr, P., Raabe, D., Zhao, X., 2013. Simulation of shear banding in heterophase co-deformation: Example of plane strain compressed $\mathrm{Cu}-\mathrm{Ag}$ and $\mathrm{Cu}-\mathrm{Nb}$ metal matrix composites. Acta Materialia 61, 4591-4606.

[29] Kalidindi, S. R., Anand, L., 1993. Large Deformation Simple Compression of a Copper Single Crystal. Metallurgical Transactions A 24A, 989-992.

[30] Kalidindi, S. R., Bronkhorst, C. A., Anand, L., 1992. Crystallographic texture evolution in bulk deformation processing of fcc metals. J. Mech. Phys. Solids 40, 537-569.

[31] Kalidindi, S. R., Duvvuru, H. K., Knezevic, M., 2006. Spectral calibration of crystal plasticity models. Acta Mater. 54, 1795-1804.

[32] Kalidindi, S. R., Knezevic, M., Niezgoda, S., S. J., 2009. Representation of the orientation distribution function and computation of first-order elastic properties closures using discrete Fourier transforms. Acta Mater. 57, 3916-3923.

[33] Kurma, S., Kurtz, S. K., 1994. Simulation of material microstructure using a 3D Voronoi tesselation: Calculation of effective thermal expansion coefficient of polycrystalline materials. Acta Metallurgica et Materialia 42, 3917-3927.

[34] Lebensohn, R. A., Tome, C. N., 1993. A self-consistent anisotropic approach for the simulation of plastic deformation and texture development of polycrystals: application to zirconium alloys. Acta Metall. Mater. 41, 2611-2624.

[35] Lebensohn, R. A., Tome, C. N., Castaneda, P. P., 2007. Self-consistent modelling of the mechanical behaviour of viscoplastic polycrystals incorporating intragranular field fluctuations. Philos. Mag. 87, 4287-4322.

[36] Misra, A., Kung, H., 2001. Deformation Behavior of Nanostructured Metallic Multilayers. Advanced Engineering Materials 3, 217-222.

[37] More, J. J., Sorensen, D. C., Hillstrom, K. E., Garbrow, B. S., 1984. The minpack project, W. J. Cowell, ed., Sources and Development of Mathematical Software Edition. Prentice-Hall.

[38] Ohser, J., Mueklich, F., 2000. Statistical analysis of microstructures in materials science. John Wiley Sons, Ltd.

[39] Roters, F., Eisenlohr, P., Hantcherli, L., Tjahjanto, D. D., Bieler, T. R., R. D., 2010. Overview of constitutive laws, kinematics, homogenization and multiscale methods in crystal plasticity finite-element modeling: Theory, experiments, applications. Acta Materialia 58, 1152-1211.

[40] Sakai, Y., Asano, T., Inoue, K., Wada, H., Maeda, H., 1991. Development of high strength, high conductivity $\mathrm{Cu}-\mathrm{Ag}$ alloys for high field pulsed magnet use. Applied Physics Letters 59, 2965-2967.

[41] Sakai, Y., Schneider-Muntau, H. J., 1997. Ultra-high strength, high conductivity Cu-Ag alloy wires. Acta materials 45, 1017-1023.

[42] Shaffer, J. B., Knezevic, M., Kalidindi, S. R., 2010. Buliding texture evolution networks for deformation processing of polycrystalline fcc metals using spectral approaches: applications to process design for targeted performance. Int. J. Plast. 26, 1183-1194.

[43] Tome, C. N., Canova, G. R., Kocks, U. F., Christodoulou, N., Jonas, J. J., 1984. The relation between macroscopic and microscopic strain hardening in fcc polycrystals. Acta Metallurgica $32,1637-1653$. 
LaTeX Source Files
Click here to download LaTeX Source Files: Article_source_file.zip

LaTeX Source Files
Click here to download LaTeX Source Files: Article_source_file.zip Click here to download LaTeX Source Files: Article_source_file.zip

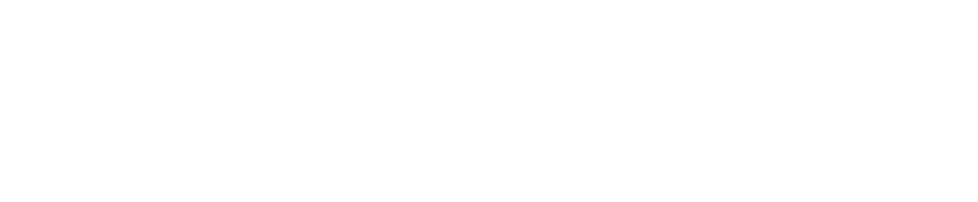

.

.

(1)

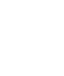

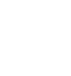

(1)

(1)

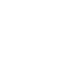

(1)

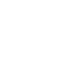

(1)

(1)

(1)

(1)

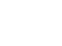

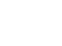

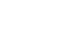

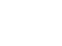

